\title{
MIMO Radar Transmit Beampattern Design with PAR Constraint in a Spectrally Crowded Environment
}

\author{
Qing-lin $\mathrm{ZHAI}^{1} *$, Yu-xi WANG ${ }^{2, *}$ and Wei $\mathrm{LI}^{2}$ \\ ${ }^{1}$ Science and Technology on Automatic Target Recognition Laboratory, National \\ University of Defense Technology, Changsha 410073, China \\ ${ }^{2}$ School of Information and Navigation, Air Force Engineering University, Xi'an 710077, \\ China \\ ${ }^{*}$ Corresponding author
}

Keywords: Transmit beampattern design, PAR, Spectral compatibility, SDR.

\begin{abstract}
Considering the fact that the electromagnetic spectrum is becoming more and more crowded due to the increasing demand for both military and civilian wireless services. A novel MIMO radar transmit beampattern design scheme with PAR constraint in a spectrally crowded environment is proposed. Different with the existing beampattern design methods, the PAR and spectral constraints are considered in the optimization of the space-time transmit waveform covariance matrix. Semidefinite relaxation (SDR) is adopted to tackle the resulting NP-hard optimization problem. With the optimized covariance matrix, the final transmit waveforms which satisfy the practical constraints can be synthesized directly via randomization. Some numerical simulation results are shown to demonstrate the effectiveness of the new technique to devise MIMO radar transmit waveforms in beampattern design complying with the PAR and spectral coexistence requirements.
\end{abstract}

\section{Introduction}

Multiple-input multiple-output (MIMO) radar as the next generation radar system has received a lot of interest in the last decade. Compared with the traditional phased array radar, MIMO radar can emit different waveforms from different transmit antennas, which can provide extra degrees-of-freedom (DOF) to improve system performance. Thus the flexible waveform design for transmit beampttern is of interest for colocated MIMO radars [1].

In order to focus the transmit power into a range of interesting angles while minimizing the transmit power for other directions, there are usually two steps to achieve this goal. In the first step, a waveform covariance matrix $\mathbf{R}$ [2] should be designed according to the desired transmit beampattern. Various criterion such as minimum mean square error (MMSE) [2-4], minimum difference (MD) [5], minimum peak side-lobe level (PSL) [6] and minimum integrated side-lobe level (ISL) [7, 8] can be used in this step to optimize the waveform covariance matrix. Besides, the covariance matrix can also be parameterized using the coordinates of a hypershere as shown in [4] and [9]. In the second step, the actual waveforms are synthesized to realize the optimized covariance matrix with a peak-to-average power ratio (PAR) or constant envelope constraint [10]. However, it was noted in $[4,10]$ that this step is not easy to achieve. A more efficient technique proposed in [11] introduces a weighting matrix to several orthogonal waveforms and optimizes the covariance matrix of weighting matrix instead of the covariance matrix of transmitted waveforms. Once the covariance matrix 
is obtained, the weighting matrix can be easily derived using randomization procedure [12].

Although many solutions and discussions have been made upon this problem, no open lecture has studied the case of MIMO radar transmit beampattern design in a spectrally crowded environment, which is an important and challenging problem due to the increasing demand for spectrum from both military sensing applications and civilian wireless services. The design of advanced radar waveforms to improve the spectral compatibility with other overlaid licensed radiators is of primary concern [13]. Recently, many papers have considered radar waveform design with a suitable frequency location [14] to mitigate interference [15] or set the signal-to-interference plus noise ratio (SINR) as the figure of merit to optimize the transmitted waveform by constraining the overall amount of interference energy on crowded frequency bands [16, 17].

In this letter, a novel optimization technique to design MIMO radar transmit beampattern while ensuring the PAR constraint of the transmit waveforms and the spectral coexistence with other overlaid radiators is proposed. Unlike the existing MIMO radar transmit beampattern design methods [2-10], through mathematical manipulations, the covariance matrix of the space-time transmit waveform can be optimized directly with the practical constraints of the maximum radiated energy on the shared bandwidth and the PAR of each antenna's transmit sequence. The resulting optimization problem is a nonconvex quadratically constrained quadratic programming (QCQP) problem which is a NP-hard problem due to the presence of constraints of the spectral compatibility and the PAR. Hence, the semidefinite relaxation (SDR) is used to solve this nonconvex QCQP problem in polynomial time. Then, with the optimized covariance matrix, the final transmit waveforms which satisfy those practical constraints can be synthesized directly through the randomization procedure [18]. Finally, some interesting case studies are analyzed to assess the performance of the proposed algorithm.

Notations: $(\cdot)^{T},(\cdot)^{*}$ and $(\cdot)^{H}$ denote the transpose, conjugate and conjugate transpose, respectively. $|\cdot|,\|\cdot\|$ and $\otimes$ denote absolute operate, Euclidean norm and the Kronecker product. $\mathbf{I}_{N}$ means $N \times N$ identity matrix. $\operatorname{vec}(\cdot)$ and $\operatorname{tr}(\cdot)$ denote vectorization operator and trace operator, respectively.

\section{System Model}

Suppose a colocated MIMO radar system with $M$ transmitters and the transmit array is assumed to be uniform linear array (ULA) with half-a-wavelength element-separation. Let

$$
\boldsymbol{s}_{m}=\left[s_{m}(0), s_{m}(1), \ldots, s_{m}(N-1)\right]^{T}
$$

denote the transmit sequence of the $m$ th antenna and $N$ is the number of samples of each transmit signal pulse. So the transmit waveform matrix of the MIMO radar is

$$
\mathbf{S}=\left[\boldsymbol{s}_{1}, \boldsymbol{s}_{2}, \ldots, \boldsymbol{s}_{M}\right]^{T} \in C^{M \times N}
$$

The steering vector of the array towards direction $\theta$ is denoted as $\boldsymbol{a}(\theta)=\left[1, e^{-j \pi \sin (\theta)}, \ldots, e^{-j(M-1) \pi \sin (\theta)}\right]^{T}$. The transmit beampattern can be defined as

$$
P(\theta) \simeq \boldsymbol{a}^{H}(\theta) \mathbf{R} \boldsymbol{a}(\theta)
$$

where $\mathbf{R} \triangleq \mathbf{S S}^{H}$ is the approximated covariance matrix using a finite number of samples. 
Due to the limited dynamic range of the radio frequency amplifier, the transmit waveform of each antenna needs to have a low PAR, which can be formulated as follows:

$$
\operatorname{PAR}\left(s_{m}\right)=\frac{\max _{n}\left|s_{m}(n)\right|^{2}}{\frac{1}{N} \sum_{n=0}^{N-1}\left|s_{m}(n)\right|^{2}} \leq \rho, \rho \in[1, N]
$$

where $\rho$ is the given upper bound to the PAR. If $\rho=1$, the transmit waveform is constant-envelope and if $\rho=N$, it means that there is no requirement on waveform's amplitude.

As to the licensed radiators coexisting with MIMO radar, suppose that each of them works over a frequency band $\Omega_{l}=\left[f_{1}^{l}, f_{2}^{l}\right], l=1,2, \ldots, L$, where $f_{1}^{l}$ and $f_{2}^{l}$ are the lower and upper normalized frequencies for the $l$ th radiator, respectively. To ensure the spectral compatibility, the transmit waveform has to be shaped to manage the amount of interfering energy produced on the shared bandwidth between licensed radiators and MIMO radar. From an analytical point of view, the energy transmitted on the $l$ th bandwidth from the $m$ th antenna is

$$
\int_{f_{1}^{l}}^{f_{2}^{l}} S_{m}(f) d f=\boldsymbol{s}_{m}^{H} \mathbf{R}_{I}^{l} \boldsymbol{s}_{m}
$$

where $S_{m}(f)=\left|\sum_{n=1}^{N} s_{m}(n) e^{-j 2 \pi f n}\right|^{2}$ is the energy spectral density (ESD) of the code $s_{m}$ and

$$
\mathbf{R}_{I}^{l}(p, q)=\left\{\begin{array}{lc}
f_{2}^{l}-f_{1}^{l} & p=q \\
\frac{e^{j 2 \pi(p-q) f_{2}^{k}}-e^{j 2 \pi(p-q) f_{1}^{k}}}{j 2 \pi(p-q)} & p \neq q
\end{array} \quad(p, q) \in\{1, \ldots, N\}^{2}\right.
$$

\section{Transmit Beampattern Design and Waveform Synthesis}

In this section, a transmit beampattern design method that optimize the covariance matrix of the transmit space-time waveform with practical constraints of spectral coexistence and PAR is introduced. To this end, let the desired beampattern denoted as $P_{d}(\theta)$, the desired power level within the focusing angle range is 1 and that outside this range is 0 . The total angle interval $\Theta=[-\pi / 2, \pi / 2]$ can be divided into $L$ grid of points denoted e.t. $\theta_{l} \in \Theta, l=1,2, \ldots, L$. The beampattern design cost function can be defined as

$$
J(\alpha, \mathbf{S})=\sum_{l=1}^{L}\left|\alpha P_{d}\left(\theta_{l}\right)-\boldsymbol{a}^{H}\left(\theta_{l}\right) \mathbf{S S}^{H} \boldsymbol{a}\left(\theta_{l}\right)\right|^{2}
$$

where $\alpha$ is a scalar factor. So the design problem can be mathematically expressed as:

$$
P \begin{cases}\min _{\alpha, \mathbf{S}} & \sum_{l=1}^{L}\left|\alpha P_{d}\left(\theta_{l}\right)-\boldsymbol{a}^{H}\left(\theta_{l}\right) \mathbf{S} \mathbf{S}^{H} \boldsymbol{a}\left(\theta_{l}\right)\right|^{2} \\ \text { s.t. } & \sum_{m=1}^{M} \boldsymbol{s}_{m}^{H} \mathbf{R}_{I} \boldsymbol{s}_{m} \leq E_{I} \\ & \frac{\max _{n}\left|s_{m}(n)\right|^{2}}{} \leq \rho, m=1,2, \ldots, M \\ & \frac{1}{N} \sum_{n=0}^{N-1}\left|s_{m}(n)\right|^{2} \\ & \operatorname{tr}\left(\mathbf{S S}^{H}\right)=E\end{cases}
$$


where $\mathbf{R}_{I}=\sum_{l=1}^{L} w_{l} \mathbf{R}_{I}^{l}, w_{l}$ is the weight coefficient associated with the lth radiator, $E$ is the total transmit power and $E_{l}$ denotes the maximum allowed interference in spectrum that can be tolerated by other radio systems. In order to solve this optimization problem, we need to do some mathematical manipulations. With the help of properties $\operatorname{vec}(\mathbf{A B C})=\left(\mathbf{C}^{T} \otimes \mathbf{A}\right) \operatorname{vec}(\mathbf{B})$ and $(\mathbf{A} \otimes \mathbf{B})(\mathbf{C} \otimes \mathbf{D})=(\mathbf{A C}) \otimes(\mathbf{B D})$, the transmit beampattern (3) can be transformed into

$$
\begin{aligned}
P(\theta) & \simeq \boldsymbol{a}^{H}(\theta) \mathbf{R} \boldsymbol{a}(\theta)=\operatorname{vec}\left(\boldsymbol{a}^{H}(\theta) \mathbf{S}\right)^{H} \operatorname{vec}\left(\boldsymbol{a}^{H}(\theta) \mathbf{S}\right) \\
& =\left(\mathbf{I}_{N} \otimes \boldsymbol{a}^{H}(\theta) \operatorname{vec}(\mathbf{S})\right)^{H}\left(\mathbf{I}_{N} \otimes \boldsymbol{a}^{H}(\theta) \operatorname{vec}(\mathbf{S})\right) \\
& =\operatorname{vec}(\mathbf{S})^{H} \mathbf{I}_{N} \otimes \boldsymbol{a}(\theta) \mathbf{I}_{N} \otimes \boldsymbol{a}^{H}(\theta) \operatorname{vec}(\mathbf{S}) \\
& =\boldsymbol{x}^{H} \mathbf{I}_{N} \otimes\left(\boldsymbol{a}(\theta) \boldsymbol{a}^{H}(\theta)\right) \boldsymbol{x} \\
& =\boldsymbol{x}^{H} \mathbf{V}(\theta) \boldsymbol{x} \\
& =\operatorname{tr}(\mathbf{V}(\theta) \mathbf{X})
\end{aligned}
$$

where $\boldsymbol{x}=\operatorname{vec}(\mathbf{S}), \mathbf{V}(\theta)=\mathbf{I}_{N} \otimes\left(\boldsymbol{a}(\theta) \boldsymbol{a}^{H}(\theta)\right)$ and $\mathbf{X}=\boldsymbol{x} \boldsymbol{x}^{H}$. Let $\boldsymbol{e}_{m}$ denote as a column vector of length $M$ with all elements zero except the $m$ th element equal to one, so the transmit power of the $m$ th antenna can be expressed as

$$
\begin{aligned}
& \sum_{n=0}^{N-1}\left|s_{m}(n)\right|^{2}=\boldsymbol{e}_{m}^{T} \mathbf{S S}^{H} \boldsymbol{e}_{m}^{*}=\operatorname{vec}\left(\boldsymbol{e}_{m}^{T} \mathbf{S}\right)^{H} \operatorname{vec}\left(\boldsymbol{e}_{m}^{T} \mathbf{S}\right) \\
& =\boldsymbol{x}^{H} \mathbf{I}_{N} \otimes \boldsymbol{e}_{m}^{*} \mathbf{I}_{N} \otimes \boldsymbol{e}_{m}^{T} \boldsymbol{x} \\
& =\boldsymbol{x}^{H}\left(\mathbf{I}_{N} \otimes \operatorname{Diag}\left(\boldsymbol{e}_{m}\right)\right) \boldsymbol{x}=\operatorname{tr}\left(\mathbf{B}_{m} \mathbf{X}\right)
\end{aligned}
$$

where $\mathbf{B}_{m}=\mathbf{I}_{N} \otimes \operatorname{Diag}\left(\boldsymbol{e}_{m}\right) \in C^{N M \times N M}$, Diag $\left(\boldsymbol{e}_{m}\right)$ indicates the diagonal matrix formed by the components of the vector $\boldsymbol{e}_{m}$. So the PAR constraint of the waveform transmitted by the $m$ th antenna (4) can be equivalently expressed as

$$
\max \left(\operatorname{diag}\left(\mathbf{B}_{m} \mathbf{X}\right)\right) \leq \frac{\rho}{N} \operatorname{tr}\left(\mathbf{B}_{m} \mathbf{X}\right)
$$

where $\operatorname{diag}\left(\mathbf{B}_{m} \mathbf{X}\right)$ indicates a vector formed by the diagonal elements of the matrix $\mathbf{B}_{m} \mathbf{X}$. As to the spectral constraint of MIMO radar transmit waveform, it can be expressed as

$$
\sum_{m=1}^{M} \boldsymbol{s}_{m}^{H} \mathbf{R}_{I} \boldsymbol{s}_{m}=\boldsymbol{x}^{H} \tilde{\mathbf{R}}_{I} \boldsymbol{x}=\operatorname{tr}\left(\tilde{\mathbf{R}}_{I} \mathbf{X}\right) \leq E_{I}
$$

where $\tilde{\mathbf{R}}_{I}=\mathbf{R}_{I} \otimes \mathbf{I}_{M}$. Finally, with the expressions (9),(11),(12), the optimization problem $P$ can be equivalently recast as

$$
P_{1} \begin{cases}\min _{\alpha, \mathbf{X}} \sum_{l=1}^{L}\left|\alpha P_{d}\left(\theta_{l}\right)-\operatorname{tr}\left(\mathbf{V}\left(\theta_{l}\right) \mathbf{X}\right)\right|^{2} \\ \text { s.t. } \quad \operatorname{tr}\left(\tilde{\mathbf{R}}_{I} \mathbf{X}\right) \leq E_{I} \\ & \max \left(\operatorname{diag}\left(\mathbf{B}_{m} \mathbf{X}\right)\right) \leq \frac{\rho}{N} \operatorname{tr}\left(\mathbf{B}_{m} \mathbf{X}\right), m=1,2, \ldots, M \\ & \operatorname{tr}(\mathbf{X})=E \\ & \mathbf{X} \geq 0 \\ & \operatorname{rank}(\mathbf{X})=\mathbf{1}\end{cases}
$$

Obviously, problem $P_{1}$ is a nonconvex problem and its nonconvexity is completely confined in the last rank constraint. So we resort the SDR framework and the SDR of problem $P_{1}$, obtained by dropping the rank-one constraint, shares the following form 


$$
P_{2} \begin{cases}\min _{\alpha, \mathbf{X}} & \sum_{l=1}^{L}\left|\alpha P_{d}\left(\theta_{l}\right)-\operatorname{tr}\left(\mathbf{V}\left(\theta_{l}\right) \mathbf{X}\right)\right|^{2} \\ \text { s.t. } & \operatorname{tr}\left(\tilde{\mathbf{R}}_{I} \mathbf{X}\right) \leq E_{I} \\ & \max \left(\operatorname{diag}\left(\mathbf{B}_{m} \mathbf{X}\right)\right) \leq \frac{\rho}{N} \operatorname{tr}\left(\mathbf{B}_{m} \mathbf{X}\right), m=1,2, \ldots, M \\ & \operatorname{tr}(\mathbf{X})=E, \mathbf{X} \geq 0\end{cases}
$$

After relaxation, problem $P_{2}$ is a semidefinite programming (SDP) convex problem and its optimal solution $\mathbf{X}^{\star}$ can be obtained with CVX toolbox in polynomial time. However the optimized covariance matrix $\mathbf{X}^{\star}$ could not be feasible to $P_{1}$, because it is not rank-one. Hence, in the following, a randomization procedure is used to synthesize the final high-quality approximate transmit waveform $x$ from $\mathbf{X}^{\star}$.

If $\mathbf{X}^{\star}$ is rank-one, the transmit waveform $\boldsymbol{x}^{\star}$ can be obtained by the eigen decomposition of $\mathbf{X}^{\star}=\boldsymbol{x}^{\star}\left(\boldsymbol{x}^{\star}\right)^{H}$. In this case, the rank-one relaxation in problem $P_{2}$ is tight and the solution $\boldsymbol{x}^{*}$ is optimal. Otherwise, a suboptimal procedure can be adopted following the proposed algorithm in [18]. Interested readers can refer to [18] for more details, we will present the synthesis algorithm in the sequel to make the letter self-contained. If $\operatorname{rank}(\mathbf{X}) \geq 2$, in order to guarantee the PAR of the transmit waveform, we can define vectors $\boldsymbol{d} \in C^{N M}$ and $\boldsymbol{d}^{-} \in C^{N M}$, whose $i$ th entry are $d(i)=\sqrt{\mathbf{X}^{\star}(i, i)}$ and $d^{-}(i)=\left\{\begin{array}{l}\frac{1}{d(i)}, \text { if } d(i)>0 \\ 1, \quad \text { if } d(i)=0\end{array}, i=1, \ldots, N M\right.$, respectively. With these two vectors $\boldsymbol{d}$ and $\boldsymbol{d}^{-}$, two diagonal matrix can be formed as $\mathbf{D}=\operatorname{Diag}(\boldsymbol{d}), \mathbf{D}^{-}=\operatorname{Diag}\left(\boldsymbol{d}^{-}\right)$. Draw $K$ random vectors $\tilde{\boldsymbol{x}}_{k}(k=1, \ldots, K)$ from the complex normal distribution $N_{C}(\boldsymbol{0}, \mathbf{C})$, where $\mathbf{C}=\mathbf{D}^{-}\left(\mathbf{X}+\left(\mathbf{I}_{N M_{t}}-\mathbf{D}^{-} \mathbf{D}\right)\right) \mathbf{D}^{-}$. Define vector $\boldsymbol{x}_{k}=\boldsymbol{d} e^{j \arg \left(\tilde{x}_{g}\right)}$, for each vector $\boldsymbol{x}_{k}$, we compute the cost function

$$
\beta_{k}=\sum_{l=1}^{L}\left|\alpha P_{d}\left(\theta_{l}\right)-\boldsymbol{x}_{k}^{H} \mathbf{V}\left(\theta_{l}\right) \boldsymbol{x}_{k}\right|^{2}
$$

and choose the minimal value over $\left\{\beta_{1}, \beta_{2}, \ldots, \beta_{K}\right\}$, the final suboptimal solution is

$$
\boldsymbol{x}^{\star}=\arg \min _{\boldsymbol{x}_{k}} \sum_{l=1}^{L}\left|\alpha P_{d}\left(\theta_{l}\right)-\boldsymbol{x}_{k}^{H} \mathbf{V}\left(\theta_{l}\right) \boldsymbol{x}_{k}\right|^{2}
$$

It worth pointing out that in the optimization stage we have already taken the spectral compatibility and the PAR constraints into account, so we don't need to synthesize the transmit waveform which has to satisfy the practical PAR constraint according to the given covariance matrix $\mathbf{R}$ as the conventional methods do. On the contrary, a high-quality transmit waveform with PAR constraint can be obtained directly through randomization procedure, which is more convenient. The number of randomizations $K$ allows to improve the approximation quality and the approximation bound is guaranteed [19]. As to the computational complexity, the computational burden is composed by two parts. The first is the complexity of solving the SDP problem $P_{2}$, which is of order $O\left((M N)^{4.5}\right)$, and the second part is the complexity of the randomization step to synthesize final waveforms, which is of order $O\left(K(M N)^{2}\right)$.

\section{Simulation Results}

We assume a ULA of $M=10$ transmit antenna elements spaced half a wavelength apart from each other. The carrier frequency and bandwidth of the transmitted waveforms are $f_{0}=10 \mathrm{GHz}$ and $B=10 \mathrm{MHz}$. The code length is $N=16$ and the total transmit energy 
is $E=1 . K=500$ is the number of randomization and the interest angle sector is $\left[-20^{\circ}, 20^{\circ}\right]$.

It is noted that, according to the expression (9), we have

$$
\boldsymbol{a}^{H}(\theta) \mathbf{R} \boldsymbol{a}(\theta)=\operatorname{tr}(\mathbf{V}(\theta) \mathbf{X})
$$

which means that the optimal beampattern is determined by the freedoms of the transmit array and the PAR and spectral constraints can't influence the shape of the optimal transmit beampattern. So the optimal beampatterns formed by $\mathbf{X}^{\star}$ are same with those formed by $\mathbf{R}$ introduced in [2] as shown in Fig. 1. And the beampatterns formed by $\mathbf{X}^{\star}$ with the same element number $M=10$ under different PAR constraints are all in full accord as shown in Fig. 2. From Fig. 2 we can also observe that the beampattern obtained by the synthesized waveform $\mathbf{S}$ through randomization under different PAR constraints is close to the desired one.

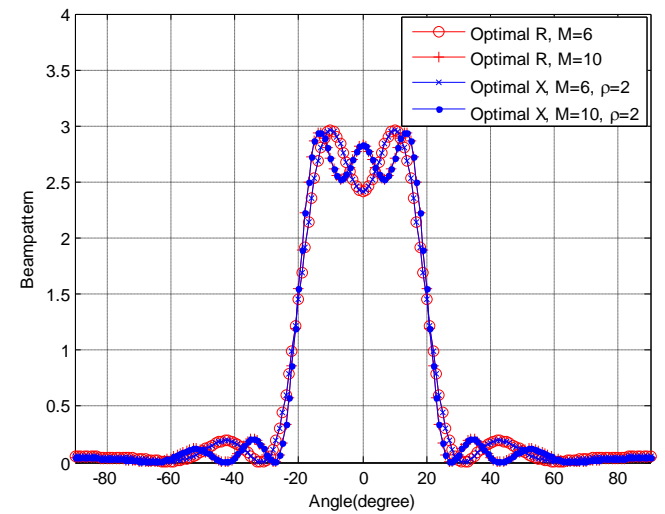

Fig. 1. Transmit Beampatterns generated by the optimal covariance matrix $\mathbf{X}^{\star}$ and $\mathbf{R}$ with different element numbers.

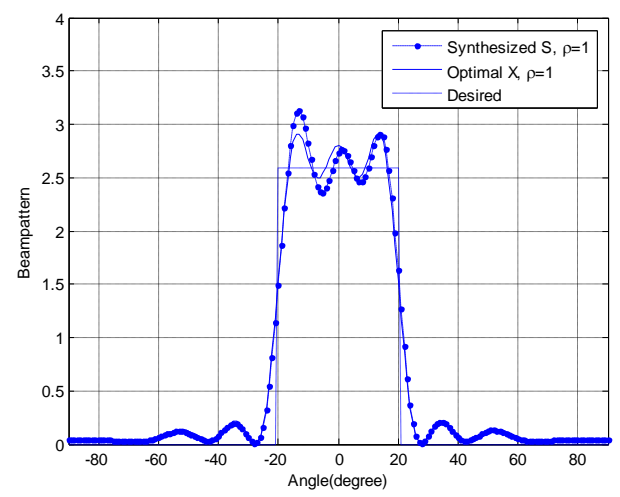

(a)

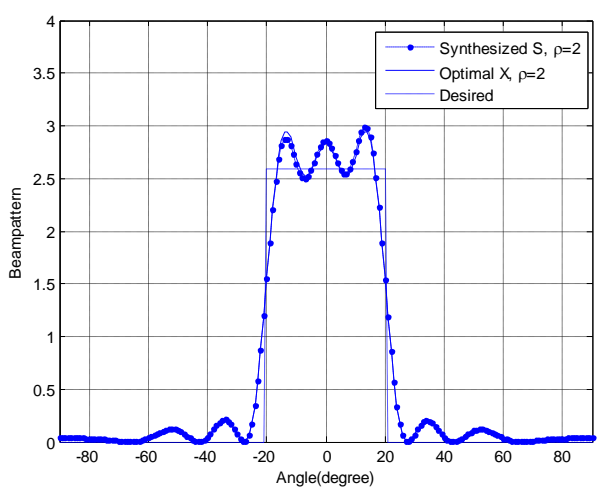

(b)

Fig. 2. Beampattern matching design with same element number $M=10$ and different PAR constraints.

According to problem $P_{2}$, we know that the optimized $\mathbf{X}^{\star}$ satisfies the PAR constraint, so the synthesized $\mathbf{S}$ via randomization also satisfies the PAR constraint as shown in Fig. 3. It is interested to observe that the PAR of the synthesized waveform through the proposed method is small even under the unconstrained case. Hence, it is reasonable to believe that the PAR of the ideal waveform whose covariance is $\mathbf{R}$ should also be small. 


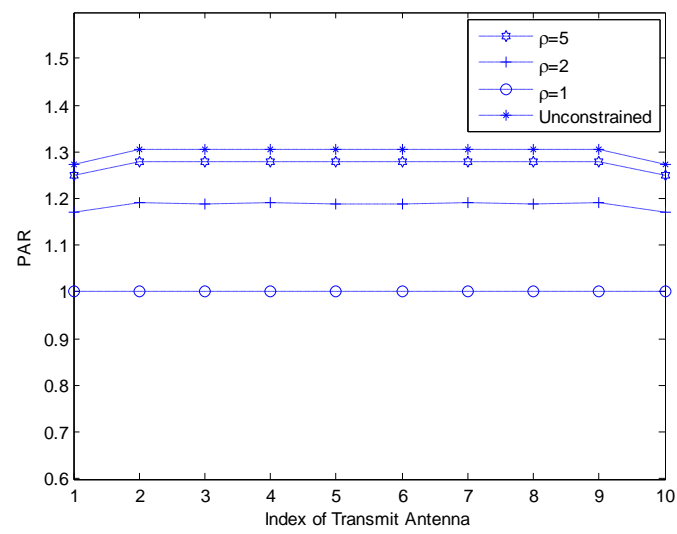

Fig. 3. Actual PAR values for the synthesized waveforms with different PAR constraints.

In order to assess the performance of the randomization procedure, we let

$$
\delta=\sum_{l=1}^{L}\left|\alpha P_{d}\left(\theta_{l}\right)-\left(\boldsymbol{x}^{\star}\right)^{H} \mathbf{V}\left(\theta_{l}\right) \boldsymbol{x}^{\star}\right|^{2}
$$

denote the difference between the beampattern formed by the synthesized waveform and the desired one. For the case of $\rho=1$, the average value of $\delta$ obtained by 100 Monte Carlo simulation runs is shown in Fig. 4 as a function of the randomization number $K$. From Fig. 4, we can see that the average value of $\delta$ decreases as $K$ increases and when $K \geq 500$, the quality of synthesized waveforms can be guaranteed.

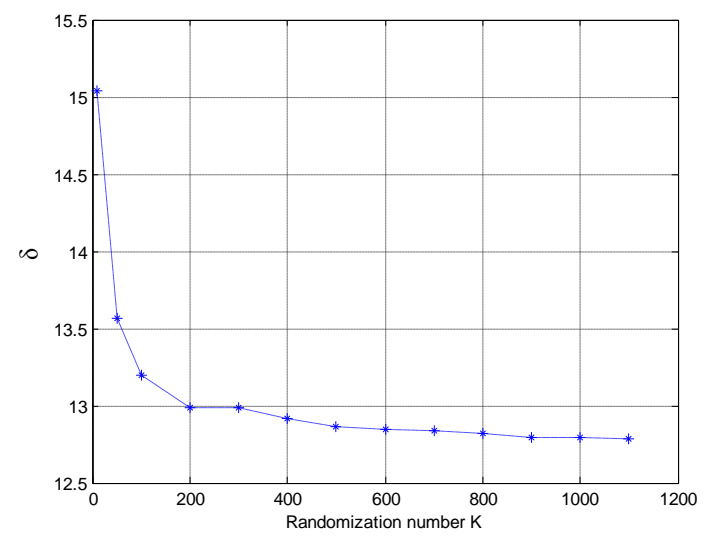

Fig. 4. Average value of $\delta$ with $\rho=1$, as a function of the randomization number K.

Finally, we extend the proposed algorithm to accommodate the spectral constraint. Suppose there are two coexisting licensed radiators and their normalized frequency bands are $\left[f_{1}^{1}, f_{2}^{1}\right]=[0.4,0.5],\left[f_{1}^{2}, f_{2}^{2}\right]=[0.8,0.9]$, respectively. Without loss of generality suppose $w_{1}=w_{2}=1$, the maximum allowed interference is $E_{I}=5 \times 10^{-4}$ and the PAR constraint is $\rho=1$. We define the total energy spectral density of MIMO radar as

$$
S(f)=\sum_{m=1}^{M} S_{m}(f)
$$

The spectral coexisting performance of the proposed method is shown in Fig. 5. It is obvious that the synthesized waveforms can control the amount of energy produced on the working frequency bands of the radiators. As a result, the spectral coexistence of the MIMO radar with other wireless system is improved. 


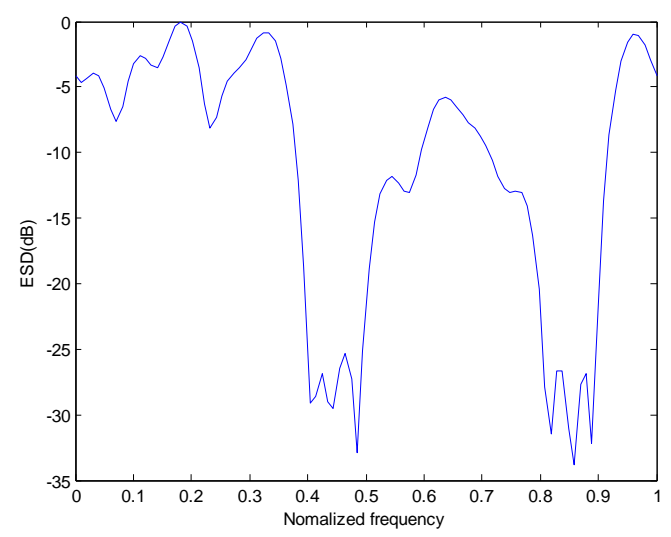

Fig. 5. ESD of the synthesized waveforms.

\section{Conclusion}

We have considered the problem of MIMO radar transmit beampattern design with a PAR constraint in a spectrally crowded environment. Different with the existing beampattern design methods, we take the practical constraints of PAR and spectral coexistence into account during the optimization of the space-time waveform covariance matrix $\mathbf{X}$. After manipulation, the new optimization problem is a QCQP nonconvex problem. In order to tackle this NP-hard problem, semidefinite relaxation framework is adopted and the optimal covariance matrix $\mathbf{X}$ can be achieved. Because the optimal $\mathbf{X}$ satisfies these constraints, the synthesized waveforms through randomization procedure also have a satisfying performance in PAR and spectral compatibility. Finally, a numerical simulation results have been provided to demonstrate the effectiveness of the proposed algorithm.

\section{References}

[1] J. Li, and P. Stoica, "MIMO radar with colocated antennas: Review of some recent work," IEEE Signal Process. Mag., vol.24, no.5, pp.106-114, Sep. 2007.

[2] P. Stoica, J. Li, and Y. Xie, "On probing signal design for MIMO radar," IEEE Trans. Signal Process., vol.19, no.2, pp. 4152-4161, Aug. 2007.

[3] D. R. Fuhrmann, G. San Antonio, "Transmit beamforming for MIMO radar systems using signal cross-correlation," IEEE Trans. Aerosp. Electron. Syst., vol.44, no.1, pp.171-186, Jan.2008.

[4] S. Ahmed, J. S. Thompson, Y. R. Petillot, and B. Mulgrew, "Finite alphabet constant-evvelope waveform design for MIMO radar," IEEE Trans. Signal Process., vol.59, no.11, pp.5326-5337, Nov. 2011.

[5] A. Khabbazibasmenj, A. Hassanien, S. A. Vorobyov, and M. W. Morency, "Efficient transmit beamspace design for search-free based DOA estimation in MIMO radar," IEEE Trans. Signal Process., vol.62, no.6, pp.1490-1500, Mar.2014.

[6] P. Gong, Z. Shao, G. Tu, and Q. Chen, "Transmit beampattern design based on convex optimization for MIMO radar systems," Signal Process., vol.94, pp.195-201, Jan. 2014.

[7] H. Xu, J. Wang, J. Yuan, and X. Shan, "MIMO radar transmit beampattern synthesis 
via minimizing sidelobe level," Progress in Electromagnetics Research B, vol.53, pp.355-371, 2013.

[8] H. Xu, R. S. Blum, J. Wang, and J. Yuan, "Colocated MIMO radar waveform design for transmit beampattern formation," IEEE Trans. Signal Process., vol.51, no.2, pp.1558-1568, Apr. 2015.

[9] S. Ahmed, J. S. Thompson, Y. R. Petillot, and B. Mulgrew, "Unconstrained synthesis of covariance matrix for MIMO radar transmit beampattern," IEEE Trans. Signal Process., vol.59, no.8, pp.3837-3849, Aug. 2011.

[10] P. Stoica, J. Li, and X. Zhu, "Waveform synthesis for diversity-based transmit beampattern design," IEEE Trans. Signal Process., vol.56, no.6, pp.2593-2598, Jan. 2008.

[11] A. Hassanien, and S. A. Vorobyov, "Transmit energy focusing for DOA estimation in MIMO radar with colocated antennas," IEEE Trans. Signal Process., vol.59, no.6, pp.2669-2682,Jun. 2011.

[12] Z. Q. LUO, W. K. MA, A. C. SO, Y. YE, and S. ZHANG, "Semidefinite relaxation of quadratic optimization problems," IEEE Signal Process. Mag., vol.27, no.3, pp.20-34, May. 2010.

[13] A. Aubry, A. De Maio, M. Piezzo, and A. Farina, "Radar waveform design in a spectrally crowded environment via non-convex quadratic optimization," IEEE Trans. Aerosp. Electron. Syst., vol.50, no.2, pp.1138-1152, Apr. 2014.

[14] M. J. Lindenfeld, "Sparse frequency transmit and receive waveform design," IEEE Trans. Aerosp. Electron. Syst., vol.40, no.3, pp.851-861, Jul. 2004.

[15] K. Gerlach, M. R. Frey, M. J. Steiner, and A. Shackelford, "Spectral nulling on transmit via nonlinear FM radar waveforms," IEEE Trans. Aerosp. Electron. Syst., vol.47, no. 2, pp.1507-1515, Apr. 2011.

[16] A. Aubry, A. De Maio, Y. Huang, M. Piezzo, and A. Farina, "A new radar waveform design algorithm with improved feasibility for spectral coexistence," IEEE Trans. Aerosp. Electron. Syst., vol.51, no.2, pp.1029-1036, Apr.2015.

[17] A. Aubry, V. Carotenuto, and A. De Maio, "Forcing multiple spectral compatibility constraints in radar waveforms," IEEE Signal Process. Lett., vol.23, no.4, pp.483-487, Apr. 2016.

[18] A. De Maio, Y. Huang, M. Piezzo, S. Zhang, and A. Farina, "Design of optimized radar codes with a peak to average power ratio constraint," IEEE Trans. Signal Process., vol.59, no.6, pp.2683-2697, Jun.2011.

[19] S. He, Z. Q. Luo, J. Nie, and S. Zhang, "Semidefinite relaxation bounds for indefinite homogeneous quadratic optimization," SIAM J. Optim., vol.18, no.2, pp.503-523, 2008. 